\title{
EFICACIA DEL PROGRAMA DE EDUCACIÓN AFECTIVO-SEXUAL EAS-SALUSEX PARA ADOLESCENTES CON TEA
}

\author{
Ma Dolores Gil-Llario \\ Salusex-Departamento de Psicología Evolutiva y de la Educación. Universitat de València \\ Dolores.gil@uv.es \\ Estefanía Ruiz-Palomino \\ Salusex-Departamento de Psicología Básica, Clínica y Psicobiología. Universitat Jaume I \\ Paula Iglesias-Campos \\ Salusex-Departamento de Psicología Evolutiva y de la Educación. Universitat de València \\ Olga Fernández-García \\ Salusex-Departamento de Psicología Evolutiva y de la Educación. Universitat de València
}

Fecha de Recepción: 6 Abril 2018

Fecha de Admisión: 10 Abril 2018

\section{RESUMEN}

Los/as adolescentes con TEA se caracterizan por carecer, en la mayoría de los casos, de las habilidades necesarias para establecer interacciones con fines afectivo-sexuales. Por este motivo, a demanda de un grupo de padres y profesores de un instituto público de Educación Secundaria, el equipo SALUSEX ha diseñado e implementado un programa de educación afectivo-sexual especialmente dirigido a satisfacer sus necesidades tanto en lo referido a conocimientos y actitudes como, sobre todo, en habilidades de comunicación e interacción con sus iguales con fines afectivo-sexuales. El programa fue aplicado a los 10 adolescentes (50\% chicos y $50 \%$ chicas) de entre 14 y 17 años que formaban parte del aula CyL de un Instituto de Educación Secundaria de Castellón de la Plana. Los resultados muestran que su nivel de conocimientos ha mejorado sustancialmente ya que, por ejemplo, el $97,2 \%$ conocen la información básica en relación con la higiene corporal y la prevención de ITS; también han mejorado sus actitudes siendo un 87,5\% quienes responden acertadamente en cuanto a la prevención del sexismo y la violencia de género, pero lo más remarcable es su mejora en cuanto a las habilidades habiendo adquirido un $90,5 \%$ unas pautas correctas de comportamiento en las relaciones de pareja. También son destacables las cifras crecientes a lo largo de las sesiones en cuanto a la motivación y satisfacción con los avances adquiridos. Se concluye la necesidad de normalizar la educación afectivo-sexual en este colectivo dada la creciente demanda y los buenos resultados obtenidos en este estudio piloto.

Palabras clave: adolescentes; TEA; educación afectivo-sexual; habilidades sociales 


\section{EFICACIA DEL PROGRAMA DE EDUCACIÓN AFECTIVO-SEXUAL EAS-SALUSEX PARA ADOLESCENTES CON TEA}

\section{ABSTRACT}

Efficacy of the eas-salusex affective-sexual education program for adolescents with asd. Adolescents with ASD are characterized by lack, in most cases, of the skills necessary to establish interactions with affective-sexual objectives. For this reason, at the request of a group of parents and teachers of a public High School, the SALUSEX team has designed and implemented a program of affective-sexual education especially aimed at satisfying their needs both in terms of knowledge and attitudes and, above all, in communication skills and interaction with their peers for affective-sexual purposes. The program was applied to the 10 teenagers (50\% boys and $50 \%$ girls) between 14 and 17 years old that were part of the CyL classroom of a High School in Castellón de la Plana. The results show that their level of knowledge has improved substantially since, for example, $97.2 \%$ know the basic information in relation to body hygiene and STI prevention; they have also improved their attitudes because $87.5 \%$ responding correctly in terms of prevention of sexism and gender violence, but the most remarkable thing is their improvement in skills, having acquired $90.5 \%$ correct guidelines for behavior in couple relationships. Also noteworthy are the increasing numbers throughout the sessions in terms of motivation and satisfaction with the progress made. The need to normalize affective-sexual education in this group is concluded given the growing demand and good results obtained in this pilot study.

Keywords: teenagers; ASD; affective-sexual education; social skills

\section{ANTECEDENTES}

La adolescencia es el periodo del ciclo vital caracterizado por múltiples cambios físicos, sociales y psicológicos. En esta etapa existe una apertura del adolescente al mundo afectivo-sexual. Los primeros contactos sexuales se producen en edades muy tempranas, convirtiéndose así en un colectivo de riesgo para numerosos problemas tales como el riesgo de infección por VIH y otras ITS, de embarazos no deseados pero, sobre todo, de abusos sexuales.

Los/as adolescentes con TEA experimentan los mismos cambios que los/as adolescentes con desarrollo normativo, no obstante, esta maduración a nivel físico no suele acompañarse de la correspondiente maduración a nivel cognitivo y social (Bekirogullari, Gulsen, \& Soyturk, 2011; Corona, Fox, Christodulu \& Worlock, 2016) por lo que requieren educación afectivo-sexual. Pero, además de las necesidades propias de cualquier adolescente, se añaden las particularidades que caracterizan a este colectivo como son el déficit en comunicación social lo que lleva a déficits en interacción social y las conductas e intereses restrictivos y repetitivos. Todas estas particularidades complican sus relaciones (Gougeon, 2010) y se concretan en: dificultad para responder de forma adecuada en las conversaciones; dificultades en la interpretación de la información no verbal; dificultades en la construcción de amistades y, sobre todo, dificultad para desarrollar relaciones satisfactorias. Dichos síntomas se presentan desde las primeras etapas del desarrollo del individuo, aunque pueden no ser evidentes hasta que las demandas sociales exceden su capacidad, e implican un deterioro significativo en áreas importantes de su funcionamiento habitual (DSM-5, APA, 2013), entre las que se encuentra el desarrollo sexual. Estos rasgos nucleares del trastorno hacen que las habilidades que suelen adquirirse de manera incidental en la mayoría de las personas, requieran una instrucción explícita en el caso de las personas con TEA, independientemente de su grado de afectación.

Por lo general, las personas con TEA necesitan este tipo de enseñanza en lo que se refiere al reconocimiento e interpretación de expresiones faciales, emociones y otras conductas no verbales, así como a las habilidades para iniciar, mantener y concluir interacciones sociales con sus pares (Hénault, 2006; Mehzabin \& Stokes, 2011; Travers y Tincani, 2010). Asimismo, suelen presentar 
dificultades a la hora de discriminar entre las conductas aceptables en contextos públicos y privados, así como un deficiente desarrollo de la Teoría de la Mente (Gabriels \& Van Bourgondien, 2007; Tissot, 2009). Algunos estudios (Hellemans, Colson, Verbraeken, Vermeiren, y Deboutte, 2007; Hénault, 2006) han puesto de manifiesto que, como consecuencia de estas dificultades, las personas con TEA suelen manifestar conductas socio-sexuales desadaptativas tales como desvestirse 0 masturbarse en lugares públicos, no respetar los límites de las relaciones interpersonales y la intimidad de otras personas, o hacer comentarios improcedentes con connotaciones de índole sexual. Además de dificultar el establecimiento de relaciones sociales y románticas significativas, los déficits en comunicación y habilidades sociales aumentan el riesgo de tener experiencias sexuales negativas, incluyendo la victimización 0 abuso sexual, por no saber diferenciar adecuadamente los comportamientos admisibles de los inadmisibles (Brown-Lavoie, Viecili \& Weiss, 2014; Gabriels, \& Van Bourgondien, 2007; Stokes, Newton \& Kaur, 2007). En vista de estos rasgos distintivos a nivel social, comunicativo y comportamental que presenta el TEA, es necesario poder ofrecer una respuesta educativa adaptada a las mismas, tanto en lo que se refiere al contenido como a la metodología de instrucción (Beddows \& Brooks, 2016; Hénault, 2006; Kalyva, 2010; Nichols \& BlakeleySmith, 2010; Travers \& Tincani, 2010; Tullis \& Zangrillo, 2013; Wolfe, Condo \& Hardaway, 2009).

Es importante destacar que entre la población general existe una idea equivocada bastante generalizada de que las personas con TEA carecen de emocionalidad siendo incapaces de vincularse afectivamente con otras personas y que, por consiguiente, no se pueden enamorar de otros y si tienen interés éste se basa exclusivamente en utilizar al otro como medio para conseguir un fin (Hellemans et al, 2007). Como consecuencia de ello, muchos de estos jóvenes no reciben una educación sexual adecuada ni en casa ni en la escuela, hecho que limita sus posibilidades de desarrollo óptimo.

En los últimos años, sin embargo, tanto los padres como los profesionales que trabajan con ellos (orientadores, profesores de pedagogía terapéutica, etc.) han ido siendo progresivamente más conscientes de sus necesidades y carencias y cada vez son más frecuentes sus peticiones de ayuda para atender a las demandas de los adolescentes con TEA tanto para mejorar su calidad de vida como para ayudarles a protegerse de los intentos de abuso sexual y de maltrato por parte de conocidos y desconocidos. En este sentido, muchos profesores afirman no contar con la formación adecuada para poder ofrecer una educación sexual diferencial que permita dar respuesta a las necesidades específicas de los jóvenes con TEA en esta materia (Kalyva, 2010).

\section{OBJETIVO}

El objetivo fue, a demanda de un grupo de padres y profesores de un centro de Educación Secundaria Obligatoria de la ciudad de Castellón, la elaboración y aplicación de un programa de educación afectivo sexual diseñado específicamente para adolescentes con Trastorno del Espectro del Autismo (TEA). Una vez elaborado y aplicado el programa nos proponemos evaluar su eficacia.

\section{PARTICIPANTES}

En el estudio participaron todos/as los/as estudiantes del aula de Comunicación y Lenguaje (CyL) de un instituto público de Castellón de la Plana diagnosticados con TEA. Fueron en total 10 participantes, siendo el 50\% chicos y el 50\% chicas de edades comprendidas entre los 14 y los 17 años.

Todos los participantes mostraban dificultades en comunicación, interacción social recíproca e identificación de emociones. Se trataba de un grupo muy heterogéneo con un $\mathrm{Cl}$ y desarrollo del lenguaje muy variable. Todos los nombres utilizados a lo largo del artículo son ficticios para preservar el anonimato de los/as participantes. 


\section{METODOLOGÍA}

A demanda de los padres y profesores del aula CyL del IES Bovalar se desarrolló un programa compuesto de 20 sesiones semanales impartidas a lo largo de dos cursos académicos, 2015-16 y 2016-17. Las sesiones tenían dos horas de duración y se impartían con una cadencia semanal durante su horario lectivo. La metodología utilizada parte de explicaciones breves que se apoyan en material gráfico (pictogramas y vídeos) con numerosas ejemplificaciones en una primera parte de las sesiones. A continuación comienzan las actividades prácticas mediante juegos de rol para finalizar con una pequeña reflexión y discusión grupal. Todas las sesiones se inician con unas breves preguntas cuyo objetivo es activar los conocimientos previos y al finalizar la sesión se pregunta por su valoración de la calidad de las actividades realizadas en la sesión así como de lo aprendido.

Al finalizar cada uno de los cursos se aplica una prueba de evaluación de conocimientos, actitudes y habilidades adquiridas tras haber sido trabajadas durante el curso.

El contenido del programa se agrupa en tres bloques:

Bloque 1: sexualidad y relaciones afectivas

1. ¿qué es la sexualidad? Dimensiones y funciones

2. Aprender a relacionarse con los demás

Bloque 2: sexualidad y conocimiento/cuidado de uno mismo

1. Conocimiento de uno mismo

2. Aprender a quererse uno mismo

Bloque 3. Sexualidad y comportamientos de autoprotección

1. Relaciones sexuales

2. Prevención abusos sexuales

En la tabla 1 se presentan los contenidos trabajados en cada sesión de los dos cursos académicos de que constó el programa.

Tabla 1. Contenidos del programa de Educación afectivo-sexual EAS-SALUSEX

\begin{tabular}{cl}
\hline Sesión & \multicolumn{1}{c}{ Contenido } \\
\hline 1 & ¿Qué es la sexualidad? \\
2 & Ámbitos de la salud sexual \\
3 & Claves para identificar y comprender la expresión de emociones: información verbal, no \\
& verbal y paraverbal \\
4 & Relaciones afectivas: compañerismo/amistad vs enamoramiento \\
5 & Estrategias para iniciar relaciones con personas desconocidas: amistad \\
6 & Estrategias para iniciar relaciones con personas desconocidas: pareja \\
7 & Pautas en las relaciones de pareja \\
8 & Aceptación de rechazo y negación de propuestas \\
9 & Estrategias para concluir relaciones de pareja a petición propia o ajena \\
10 & Cómo interpretar correctamente los mensajes provenientes de las redes sociales. \\
11 & Higiene y prevención de ITS \\
12 & Cambios corporales y desarrollo de la imagen corporal \\
13 & Autoestima y prevención del abuso sexual y la violencia de género \\
14 & Desarrollo y aceptación de la orientación sexual \\
15 & Prácticas sexuales: sexualidad en solitario y de pareja \\
16 & Potenciación de la autoestima y autoaceptación de la orientación sexual \\
17 & Habilidades y pautas de las relaciones de pareja \\
18 & Riesgos de las relaciones de pareja: sexting y grooming \\
19 & Prevención del sexismo, abuso sexual y violencia en las relaciones de pareja \\
20 & Prevención de comportamientos sexuales de riesgo \\
\hline
\end{tabular}




\section{RESULTADOS:}

Tras la aplicación del programa de educación afectivo-sexual EAS-TEA-SALUSEX para estudiantes con TEA a lo largo de los cursos 20015-16 y 2016-17 ofrecemos algunos indicadores del impacto del programa una vez finalizado.

1. Grado de motivación

Durante el desarrollo de las sesiones de intervención se iba tomando registro de su grado de participación a través de la realización de preguntas, algunas de las cuales eran pertinentes y versaban acerca de los contenidos que se estaban trabajando mientras que otras no lo eran. Mediante el registro y observación de las preguntas pertinentes de los estudiantes obtenemos información sobre el grado de motivación. En la gráfica 1 y 2 se muestra la evolución de la motivación de los participantes durante los dos cursos:

Gráfico 1. Número de preguntas y respuestas sobre los temas tratados en cada sesión del 1er curso

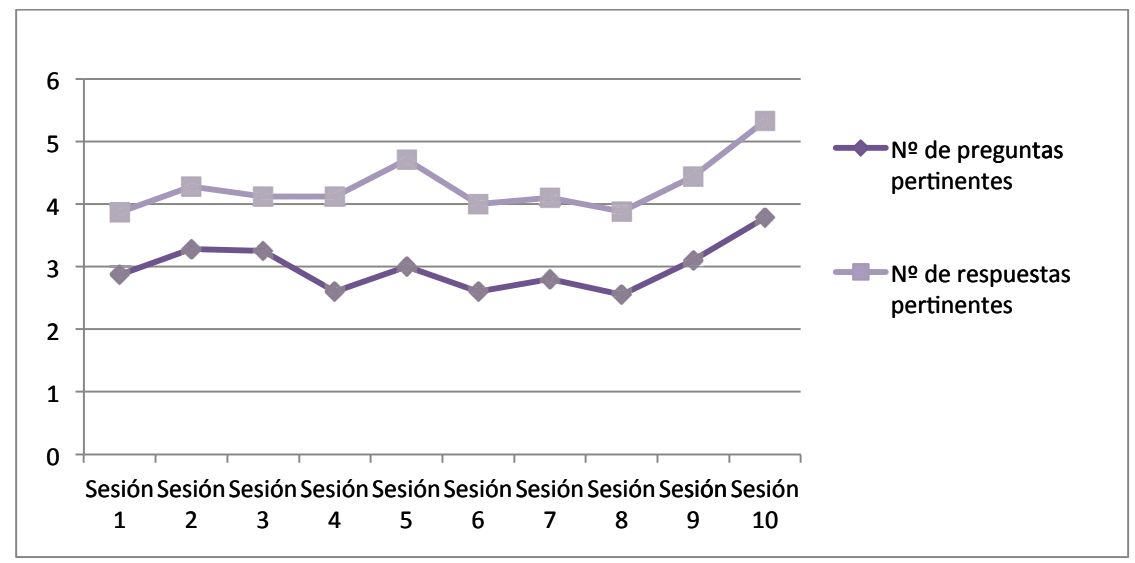

Como puede observarse, a pesar de que la participación del grupo se ha mantenido bastante estable a lo largo del programa, se ha producido un aumento considerable de la misma en las últimas sesiones tanto por el número de preguntas como de respuestas pertinentes que han formulado los participantes en general. Así, vemos un despunte de la participación en la sesión 5 explicado posiblemente por el interés del grupo en el contenido que se trató en ella (prácticas sexuales), y un despunte final ocasionado seguramente por el aumento de la confianza de los/as participantes. Al mismo tiempo, se puede observar que su participación es mayor cuando se les instiga a responder que cuando ellos/as deben ser quieres inicien la interacción (hagan las preguntas). 
Gráfico 2. Número de preguntas y respuestas sobre los temas tratados en cada sesión del $2^{\circ}$ curso

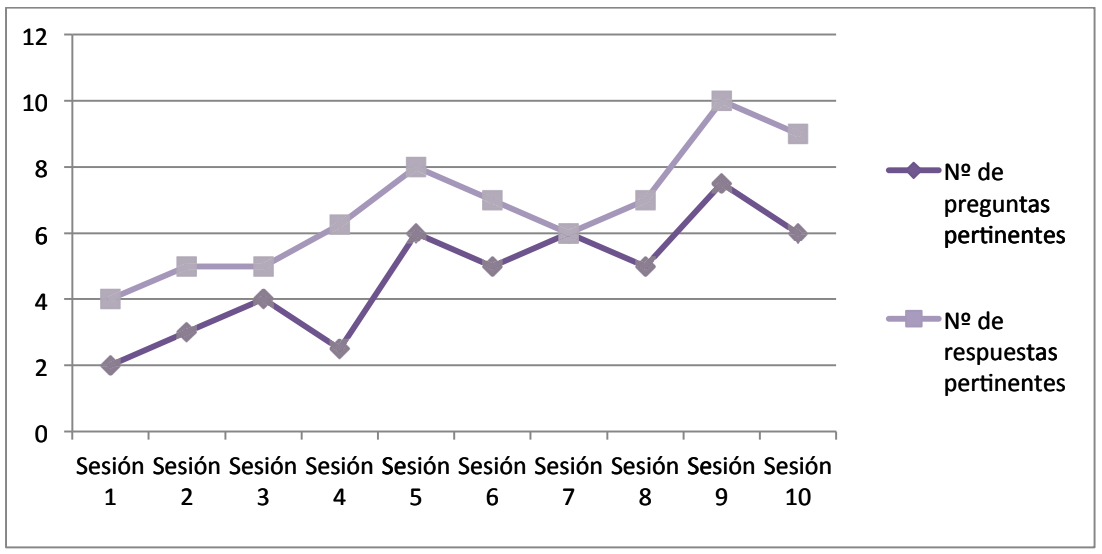

Por otro lado, los/as adolescentes han diferido en cuanto a su grado de motivación durante la aplicación del programa como podemos ver en los gráficos 3, y 4 en los que se muestra la media de preguntas y respuestas pertinentes que, de forma global, ha realizado cada participante a lo largo del programa.

Gráfico 3. Media de preguntas y respuestas de cada participante en el 1er curso

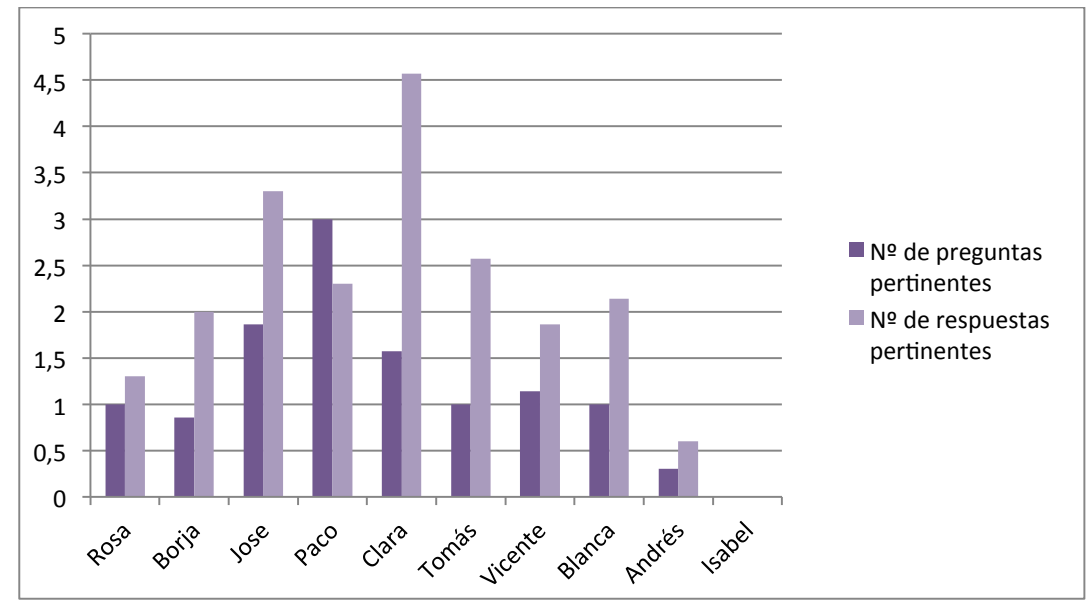


Gráfico 4. Media de preguntas y respuestas de cada participante en el $2^{\circ}$ curso

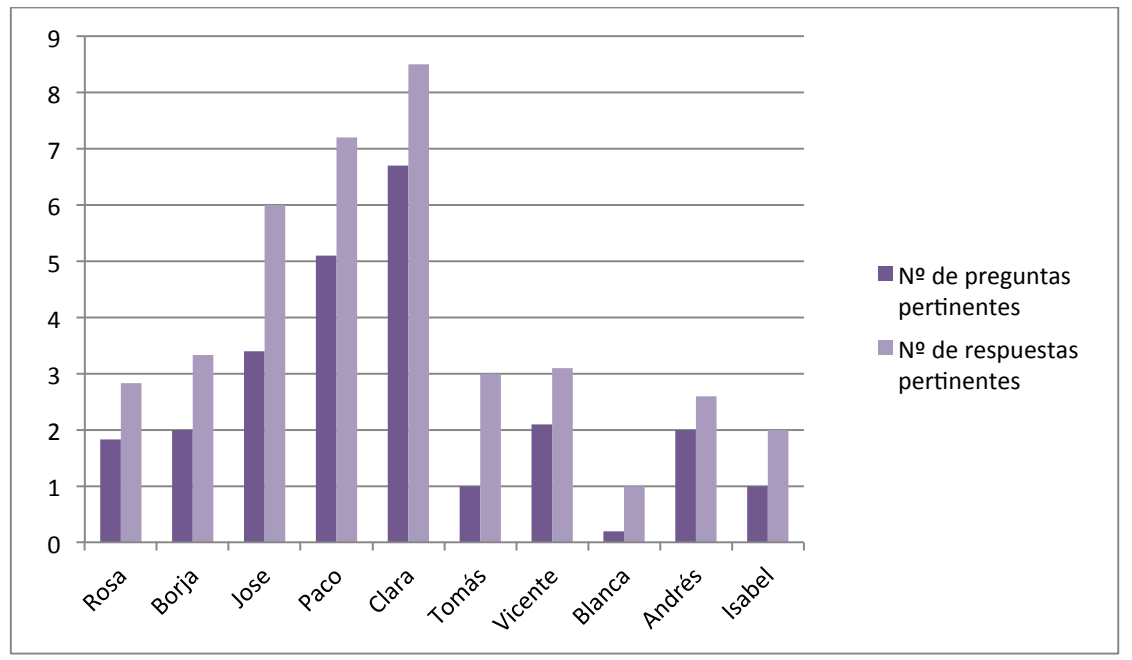

Como se puede observar el número de preguntas pertinentes correlaciona bastante con el número de respuestas pertinentes en cada participante, y que hay, además, una tendencia a que sea mayor el número de respuestas pertinentes que dan al número de preguntas pertinentes en todos los participantes. Más allá de esta tendencia general, existen diferencias individuales ya que hay adolescentes que intervenían más en las dinámicas de las sesiones (Jose, Paco, Clara) que otros (Blanca, Isabel). Estos resultados pueden deberse en parte a las diferencias en cuanto al nivel intelectual de los jóvenes, pero también a la comodidad percibida entre los miembros del grupo así como el tema del programa. Es importante resaltar que los adolescentes que muestran mayor motivación para participar también se caracterizan por mostrar un mayor rendimiento y grado de aprendizaje que aquellos que intervenían menos.

2. Grado de satisfacción:

Un segundo aspecto analizado ha sido el grado de satisfacción que se ha evaluado a partir de las valoraciones que los participantes rellenaban al finalizar cada sesión en las cuales reflejaban su percepcion subjetiva de aprendizaje y la medida en que les parecía interesante el taller. Con estos indicadores hemos obtenido información acerca del grado de satisfacción subjetiva con las sesiones en particular y con el programa en general.

En términos generales apreciamos que el grado de satisfacción de los chicos y chicas ha sido muy elevado, ya que se han encontrado cómodos tanto con la temática tratada, como con las actividades realizadas y con las monitoras que impartían el taller valorando positivamente los contenidos tratados en las sesiones, así como las dinámicas. Hay que subrayar que se ha producido un cambio importante respecto a la participación e implicación de los jóvenes a lo largo de las sesiones debido en gran parte a que la confianza con las psicólogas ha sido progresivamente mayor creando un ambiente más seguro y a que se ha incrementado la sintonía a la hora de explicar los conceptos ajustando el tipo de lenguaje al ir conociendo cada vez mejor el nivel de los participantes y su grado de comprensión o forma de llegar a los conceptos. Esto puede haber influido directamente en la percepción subjetiva de aprendizaje de los participantes, ya que manifiestan que en todas 
las sesiones han aprendido algo que no sabían siendo capaces de identificarlo (aunque se haga uso de lluvia de ideas para ayudarles en la detección).

Esta elevada satisfacción ha podido verse reflejada en el grado de participación en las actividades propuestas en las sesiones por parte de los chicos y chicas (ver gráfico 5). Ésta ha sido bastante alta y les ha permitido poner en práctica los conocimientos adquiridos. Sin embargo, dicha participación no ha sido homogénea entre los diferentes adolescentes, ya que había diferencias dependiendo de la motivación general que despertaba el programa. En ningún caso se ha hecho uso de incentivos externos, haciéndose de forma totalmente voluntaria.

También ha recibido una valoración positiva la duración de las sesiones, aunque a algunos de ellos les han parecido cortas y piensan que debían haber durado algo más.

\section{Gráfico 5. Satisfacción de los/as participantes}

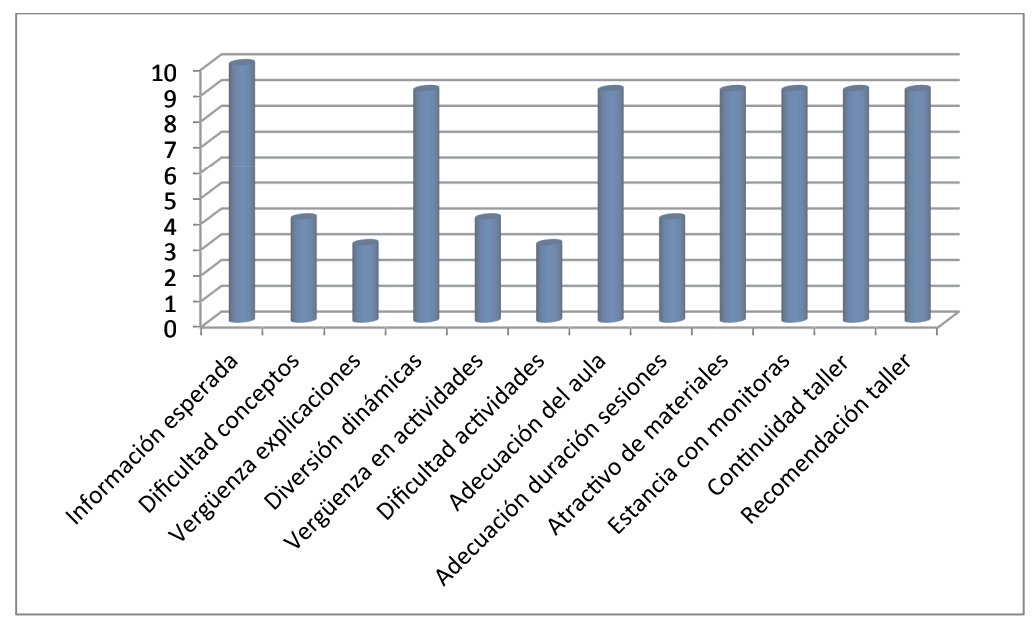

3. Grado de aprovechamiento:

Para valorar el grado de aprovechamiento de las sesiones y del programa, así como la cantidad de conocimientos y conceptos que han adquirido los estudiantes tanto de forma individual como grupal, se han valorado el número de respuestas correctas que dieron los/as alumnos/as en las cuestiones que se planteaban al inicio de cada sesión en comparación con las respuestas que dieron a estas mismas preguntas al finalizar el programa. Así, los porcentajes de aciertos obtenidos como grupo fueron los siguientes (ver gráfico 6): 
Gráfico 6. Grado de aprendizaje grupal comparando los conocimientos antes y después de la aplicación del programa en el $2^{\circ}$ curso

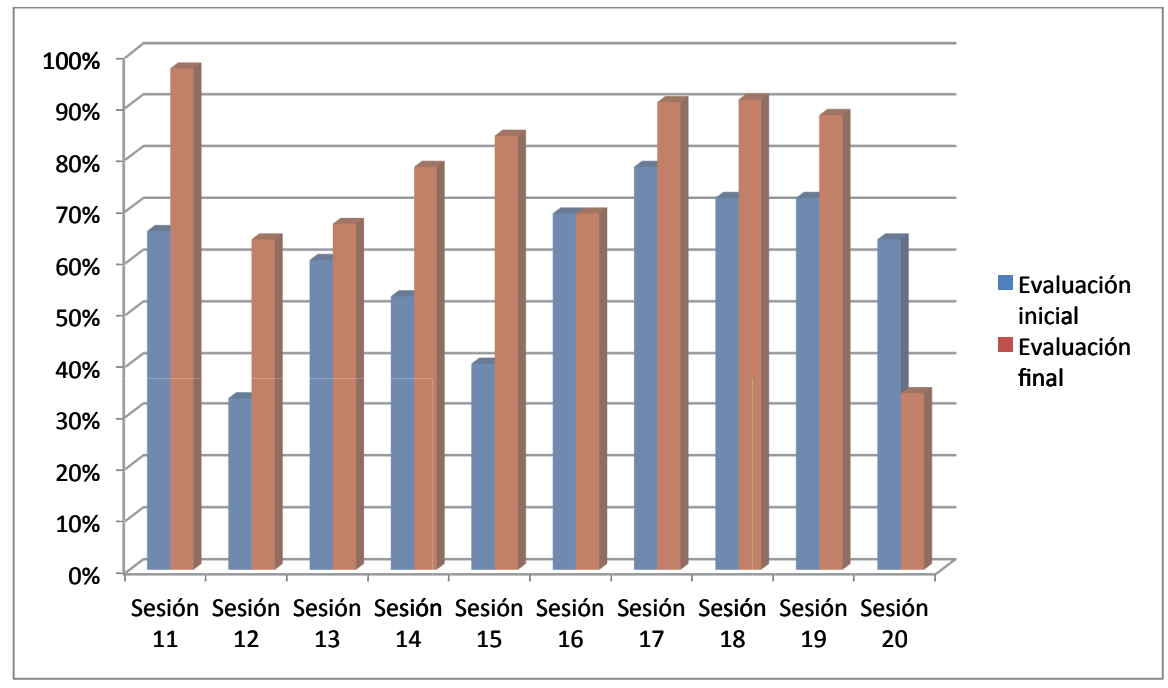

Por un lado, si comparamos los porcentajes de aciertos obtenidos por el grupo en las preguntas de las diferentes sesiones del segundo curso antes y después del programa podemos comprobar que se ha producido un aumento de los conocimientos de todas las sesiones excepto en el caso de los conocimientos sobre prevención de comportamientos sexuales de riesgo (sesión 20). Al mismo tiempo, se ha producido mayor consolidación de los conceptos relativos a la higiene (sesión 11), a las habilidades y pautas de las relaciones de pareja y contacto por redes sociales (sesión 17 y 18) y a la prevención del sexismo (sesión 9). Por otro lado, hay una serie de conocimientos que han aumentado exponencialmente tras la realización de los talleres, son los relativos a la sexualidad propia y mutua (p.ej. masturbación, sexo oral, etc.) (Sesión 15), a la higiene sexual (sesión 11), y a los cambios corporales, imagen corporal y autoestima (sesión 12).

Por otro lado, fijándonos únicamente en los contenidos con los que han finalizado el programa podemos comprobar que la mayoría de los participantes conoce los conceptos que se trabajaron sobre higiene y prevención de ITS $(97,2 \%)$, sobre habilidades y pautas de las relaciones de pareja y contacto por redes sociales (p. ej. sexting y grooming) $(90,5 \%)$ y sobre prevención del sexismo y violencia en las relaciones de pareja $(87,5 \%)$. En el otro extremo, encontramos que muy pocos han asentado conocimientos relativos a los métodos de prevención de los comportamientos sexuales de riesgo (p. ej. uso del preservativo, anticonceptivos, etc.) (34,3\%). Por otro lado, encontramos que un elevado número de participantes, aunque no la mayoría, ha adquirido conocimientos sobre los cambios corporales y la imagen corporal $(63,8 \%)$, sobre la prevención del abuso sexual $(66,6 \%)$ y la potenciación del autoestima $(68,7 \%)$. Y que un número más elevado de alumnos manifiesta poseer estrategias sobre la sexualidad propia y mutua (p. ej. qué es la masturbación o sexo oral) (84,4\%) y sobre el desarrollo y aceptación de la identidad sexual $(77,7 \%)$. Los adolescentes difieren entre sí (ver gráfico 7) en el grado de adquisición de conocimientos. 


\section{EFICACIA DEL PROGRAMA DE EDUCACIÓN AFECTIVO-SEXUAL EAS-SALUSEX PARA ADOLESCENTES CON TEA}

Gráfico 7. Grado de aprendizaje individual comparando los conocimientos antes y después de la aplicación del programa

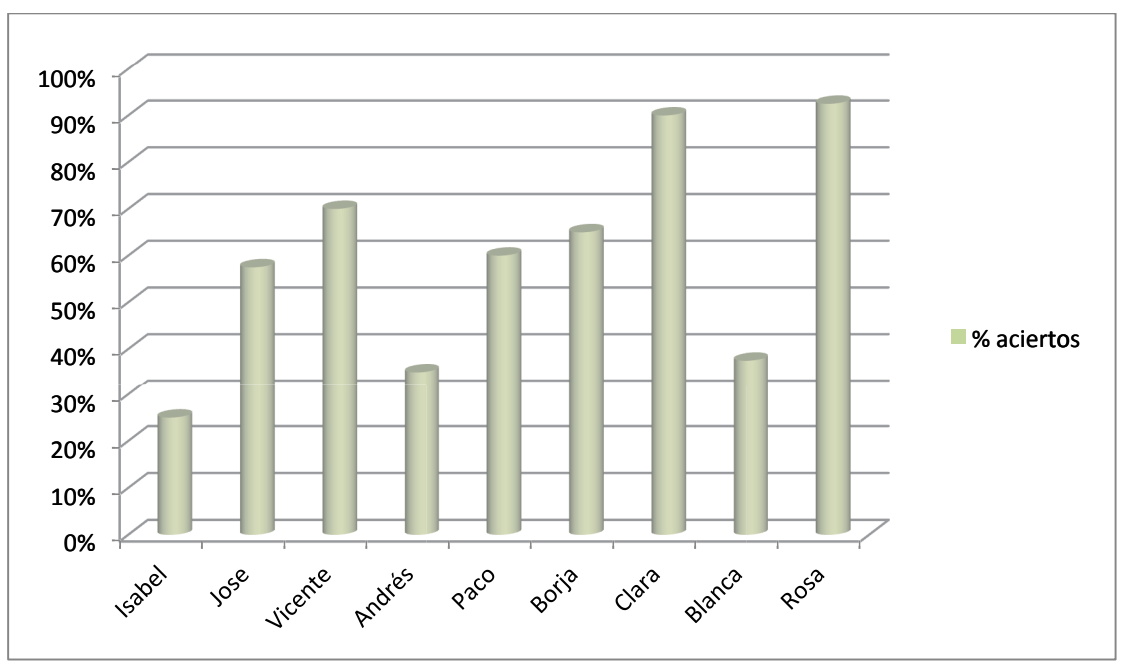

Como podemos observar, la mayoría de los participantes (un 66,6\%) ha asentado más del $50 \%$ de los conocimientos propuestos en el programa. En esta línea, cabe hacer referencia expresa a la correlación que parece mostrar los resultados con el cociente intelectual y diagnóstico al que tuvimos acceso desde el centro.

Por lo que respecta a la metodología usada, los participantes han expresado mediante las valoraciones finales de cada sesión que les parecía adecuada aunque la parte de la sesión que mayor interés les despertaba era aquella que era más práctica y en la que se llevaba a cabo la visualización de videos, el trabajo con pictogramas, diálogo crítico por grupos, o lectura de cuentos didácticos. En cuanto a las estrategias que consideran mejores para obtener los conocimientos necesarios destacan aquellas en las que se hace uso de cuentos didácticos (sesión 1 y 7 ) o la visualización de videos (sesión 8).

\section{DISCUSIÓN Y CONCLUSIONES}

El programa de educación afectivo-sexual EAS-SALUSEX-TEA ha mostrado unos resultados muy satisfactorios tanto en lo referido a la motivación despertada en el colectivo de adolescentes con TEA como en el grado de satisfacción pero sobre todo en cuanto a su capacidad para mejorar los conocimientos, actitudes y habilidades del grupo. Efectivamente, por lo que se refiere a los adolescentes se constata un elevado interés y motivación, se produjo un cambio de actitudes, una elevada adquisición de conocimientos y habilidades. Es importante subrayar la implicación activa en las actividades, si bien hay que resaltar que se pusieron de manifiesto importantes diferencias entre los participantes debidas, en gran parte, a su nivel de implicación personal determinado en gran medida por sus capacidades cognitivas. El número de preguntas y respuestas pertinentes fue aumentando progresivamente a lo largo de los dos cursos que duró el programa en la medida en que los participantes se iban familiarizando con la metodología de trabajo así como con las monitoras que, a su vez, según iban conociéndolos mejor, iban siendo cada vez más capaces de adecuar el lenguaje a las capacidades de cada participante optimizando así su rendimiento. 
El progreso y resultados del programa también fueron puestos a consideración de los padres. Hemos de recordar que el programa se desarrolló precisamente a demanda suya lo que muestra una elevada implicación. Este interés ha propiciado la facilitación de información relativa a las características individuales, aspecto que fue decisivo tanto para la elaboración inicial del programa en un intento por responder a sus necesidades específicas, como para el continuo ajuste que se ha ido realizando en cuanto a la temporalización, desarrollo y tipo de actividades que se llevaban a cabo. En todo momento han animado a los adolescentes a asistir y participar superando las reticencias iniciales. La comunicación con ellos ha sido fluida en todo momento y, por todos estos aspectos, los padres han realizado una valoración muy positiva del programa.

En tercer lugar, si atendemos a los profesionales del centro, hay que subrayar que han constituido el tercer fundamento del programa ya que han facilitado en todo momento la información relativa a las características individuales de los menores, desde su percepción profesional, lo cual ha sido decisivo en la adecuación del programa a sus necesidades. También ha sido destacable la búsqueda de horarios compatibles con el resto de sus actividades en el centro facilitando en todo momento los materiales e infraestructura necesarios. También por parte de los profesionales cabe destacar la valoración positiva que realizaron del desarrollo del programa.

Por último, en nuestra opinión, el programa ha satisfecho notablemente nuestras expectativas y nos ha dado la oportunidad de ir ajustando gracias a la colaboración de adolescentes, padres y profesionales los contenidos y la metodología del programa Entendemos que se ha logrado la mayoría de los objetivos propuestos, respondiendo de este modo en gran medida a la necesidad que presentaban los participantes al inicio del programa.

Como conclusión general, podemos decir que, tal y como señalan diferentes estudios (Kalyva, 2010, Travers \& Tincani, 2010) las perspectivas tanto de aquellos que tienen una estrecha relación con personas con TEA, con independencia del grado de afectación, como de los propios implicados convergen en que resulta necesario proporcionar una educación socio-sexual que se dirija de forma específica y significativa a las facetas en las que estas personas han manifestado tener mayores carencias y que un programa como el EAS-SALUSEX-TEA se ha mostrado suficientemente eficaz para atender dichas necesidades.

\section{RFERENCIAS BIBLIOGRAFICAS}

Beddows, N. \& Brooks, R. (2016) Inappropriate sexual behaviour in adolescents with autism spectrum disorder: what education is recommended and why. Early Intervention in Psychiatry, 10, 282-289.

Bekirogullari, Z., Gulsen, C. \& Soyturk, K. (2011). The information and attitude levels of the educational psychologists and special education teachers in the process of sex education for the adolescents with autism. Procedia, Social and Behavioral Sciences, 12, 638-653.

Brown-Lavoie, S. M., Viecili, M. \& Weiss, J. (2014) Sexual knowledge and victimization in adults with autism spectrum disorders. Journal of Autism and Developmental Disorders, 44 (9) 2185 $-2196$.

Corona, L. L., Fox, S. A., Christodulu, K. V., \& Worlock, J. A. (2016). Providing education on sexuality and relationships to adolescents with autism spectrum disorder and their parents. Sexuality and Disability, 34 (2), 199-214.

Gabriels, R. L. y Van Bourgondien, M. E. (2007). Sexuality and Autism: Individual, Family, and Community Perspectives and Interventions. En Gabriels, R. L. \& Hill, D. E. (Eds.), Growing Up with Autism Working with School-Age Children and Adolescents (pp. 58-72). New York: The Guilfrod Press. 
Gougeon, N. (2010) Sexuality and Autism: A Critical Review of Selected Literature Using a SocialRelational Model of Disability. American Journal of Sexuality Education, 5(4), 328-361.

Hellemans, H., Colson, K., Verbraeken, C., Vermeiren, R., \& Deboutte, D. (2007). Sexual behaviour in high-functioning male adolescents and young adults with autism spectrum disorder. Journal of Autism and Developmental Disorders, 37 (2), 260-9.

Hénault, I. (2006). Asperger's syndrome and sexuality: From adolescence through adulthood. Londres: Jessica Kingsley Publishers.

Kalyva E. (2010). Teachers' perspectives of the sexuality of children with autism spectrum disorders. Research in Autism Spectrum Disorders, 4 (3), 433-437.

Mehzabin, P. \& Stokes, M. (2011). Self-assessed sexuality in young adults with high-functioning autism. Research in Autism Spectrum Disorders, 5(1), 614-621.

Nichols, S. \& Blakeley-Smith, A. (2010) "I'm Not Sure We're Ready for This ...": Working With Families Toward Facilitating Healthy Sexuality for Individuals With Autism Spectrum Disorders. Social Work in Mental Health, 8(1), 72-91.

Stokes, M., A., Newton, N., \& Kaur, A. (2007). Stalking, and social and romantic functioning among adolescents and adults with autism spectrum disorder. Journal of Autism and Developmental Disorders, 37, 1969-1986.

Tissot, C. (2009). Establishing a sexual identity: Case studies of learners with Autism and learning difficulties. Autism, 13 (6), 551-566.

Travers, J. \& Tincani, M. (2010). Sexuality education for individuals with autism spectrum disorders: Critical issues and decision making guidelines. Education and Training in Autism and Developmental Disabilities, 45(2), 284-293.

Tullis, C. A., \& Zangrillo, A. N. (2013). Sexuality education for adolescents and adults with autism spectrum disorders. Psychology in the Schools, 50 (9), 866-875.

Wolfe, P. S., Condo, B., \& Hardaway, E. (2009). Sociosexuality education for persons with autism spectrum disorders using principles of applied behavior analysis. Teaching Exceptional Children, 42 (1), 50-61. 